\title{
Melting of orientational degrees of freedom
}

\author{
A. Aznar, P. Lloveras ${ }^{\mathrm{a}}$, M. Barrio, and J.Ll. Tamarit \\ Departament de Física, ETSEIB, Universitat Politècnica de Catalunya, Diagonal 647, \\ Barcelona, 08028 Catalonia, Spain
}

Received 11 July 2016 / Received in final form 22 August 2016

Published online XX XXX 2016

\begin{abstract}
We use calorimetry and dilatometry under hydrostatic pressure, X-ray powder diffraction and available literature data in a series of composition-related orientationally disordered (plastic) crystals to characterize both the plastic and melting transitions and investigate relationships between associated thermodynamic properties. First, general common trends are identified: (i) The temperature range of stability of the plastic phase $T_{m}-T_{t}$ (where $T_{t}$ and $T_{m}$ are the plastic and melting transition temperatures, respectively) increases with increasing pressure and (ii) both the rate of this increase, $d\left(T_{m}-T_{t}\right) / d p$, and the entropy change across the plastic transition analyzed as function of the ratio $T_{t} / T_{m}$ are quite independent of the particular compound. However, the dependence of the entropy change at the melting transition on $T_{t} / T_{m}$ at high pressures deviate from the behavior observed at normal pressure for these and other plastic crystals. Second, we find that the usual errors associated with the estimations of second-order contributions in the Clausius-Clapeyron equation are high and thus these terms can be disregarded in practice. Instead, we successfully test the validity of the Clausius-Clapeyron equation at high pressure from direct measurements.
\end{abstract}

\section{Orientationally disordered phases are mesophases characterized by the presence orien- tational dynamic disorder that may arise in molecular crystals constituted by rather isometric or small molecules linked by weak interactions. Activation of the orienta- tional degrees of freedom from the completely ordered crystalline phase occurs across a first-order transition with unusual large enthalpy and entropy changes that exceed those associated with the melting towards the liquid phase [1,2]. This has made them promising as solid-state thermal energy storage materials $[3,4]$. \\ Beyond this fundamental picture, several studies have been devoted to identify additional universal thermodynamic behavior [5-9], but with limited success. Mostly, the detected common trends are restricted to a given family of composition-related compounds only, rather than to actually general features within plastic crystals. \\ Interestingly, several thermodynamic correlations were noticed to be shared by a wide variety of plastic crystals [9,10]: (i) The temperature range of stability of the}

\footnotetext{
${ }^{a}$ Corresponding author: e-mail: pol.1loveras@upc.edu
} 
plastic phase, $T_{m}-T_{t}$, (where $T_{m}$ and $T_{t}$ stand, respectively, for the temperature at the melting and plastic phase transition at a given pressure) increases at the expense of the liquid phase when the pressure is increased, i.e. $d T_{m} / d p>d T_{t} / d p>0$. (ii) The entropy and volume changes at $T_{m}, \Delta S_{m}$ and $\Delta V_{m}$ respectively, decrease with pressure. These features have been explained in terms of the relative magnitude between the rotational and diffusional energy barriers [9]. On the one hand, the existence of the plastic transition occurs provided that the activation energy of rotational degrees of freedom is low enough compared to diffusion activation. On the other hand, the latter increases more rapidly with pressure than the former, which is intuitive as pressure favors the more compact stacking. In turn, a fast increase of $T_{m}$ with pressure leads to a decrease of $\Delta S_{m}$. The decrease of $\Delta V_{m}$ with pressure can then be inferred from the Clausius-Clapeyron equation, as experimental observations indicate that. Moreover, by analyzing $\Delta S_{m}$ as function of $T_{t} / T_{m}$ at normal pressure for different sets of materials, it was obtained that $\Delta S_{m}$ decreases with $T_{t} / T_{m}$, and in the limit of $T_{t} \rightarrow T_{m}, \Delta S_{m} \rightarrow R \ln 2$. A deviation from this behavior was observed, however, in a compound that differed from the rest in the nature of the intermolecular bonds [10].

It is clear that a full thermodynamic characterization of a $p V T$ system requires the knowledge of the effect of pressure on the thermodynamic variables. However, to date most high-pressure theoretical and experimental studies are rather limited and have focused mainly in the determination of the $T$ - $p$ diagram whereas high-pressure energetic and volume data are scarce, basically due to the technical difficulties and lack of standard commercial high-pressure equipment [11-19]. Clausius-Clapeyron is then evaluated at normal pressure. Moreover, investigations have been focused on inorganic and metallic compounds whereas organic plastic phases and the associated melting of orientational degrees of freedom have deserved little attention from the high-pressure community. It is worth mentioning here recent studies [20,21] on organic crystals that have suggested that application of pressure could lead to the emergence of stronger bonds between $\mathrm{C}$ atoms belonging to different molecular planes that are usually stabilized through van der Waals forces or hydrogen bonds. Applied pressures were, however, much larger than those of the present work.

Here we report systematic high-pressure experimental data on the solid and liquid phases and related transitions in a series of composition-related plastic crystals, by means of calorimetry and dilatometry under applied hydrostatic pressure and X-Ray powder diffraction. In the first part of the present study, we use these data to check and extend the aforementioned thermodynamic correlations to an unexplored range of materials and pressures.

On the other hand, the traditional Clausius-Clapeyron equation for first-order phase transitions renders a simple relation between volume and entropy changes at the transition point, $d T / d p=\Delta V / \Delta S$. Close to the transition, first-order derivatives arise and other additional relations can be derived [22]:

$$
\frac{d T}{d p}=\frac{\Delta V_{0}+\Delta \alpha_{0}^{*}\left(T-T_{0}\right)-\Delta \beta_{0}^{*}\left(p-p_{0}\right)}{\Delta S_{0}+\Delta C_{p 0} \log \left(T / T_{0}\right)-\Delta \alpha_{0}^{*}\left(p-p_{0}\right)}
$$

$$
\frac{d^{2} T}{d p^{2}}=-\frac{1}{\Delta V} \frac{d T}{d p}\left[\frac{\Delta C_{p}}{T}\left(\frac{d T}{d p}\right)^{2}-2 \Delta \alpha^{*} \frac{d T}{d p}+\Delta \beta^{*}\right]
$$

$$
\frac{d \Delta H}{d p}=\frac{d T}{d p} \Delta C_{p}+\Delta V-T \Delta \alpha^{*}
$$

$$
\frac{d \Delta V}{d p}=\frac{d T}{d p} \Delta \alpha^{*}-\Delta \beta^{*}
$$


where $C_{\mathrm{p}}$ is the specific heat capacity, and $\alpha^{*}=(\mathrm{dV} / \mathrm{dT})_{\mathrm{p}}$ and $\beta^{*}=-(\mathrm{dV} / \mathrm{dp})_{\mathrm{T}}$ are related to isobaric thermal expansion and isothermal compressibility, although the lack of the $1 / V$ factor recommends a star superscript to avoid confusion. In turn, the increments $(\Delta)$ refer to the difference between the quantities in either side of the transitions and the zero subscript (0) to the normal pressure condition. Equation (1) should then be approximately valid only in a $T$ - $p$ range close to normal pressure where the quantities appearing in the right hand side of the equation are considered to be constant. Strictly at normal pressure, Clausius-Clapeyron relation is recovered.

These equations have been largely disregarded, basically due to the lack of data, so that studies addressing them are very rare [23]. Therefore, the second part of the present work is aimed at taking advantage of the compilation of own and literature data to (i) check the traditional Clausius-Clapeyron equation evaluated at high pressure, and (ii) to obtain orders of magnitude for the above quantities and related Equations (1-4).

\section{Samples and experimental methods}

Among plastic crystals, here we focus on a series of alcohol, amine and nitrite

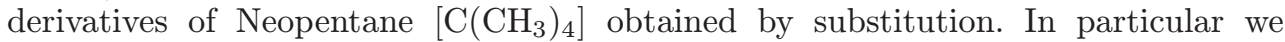
study neopentyl alcohol [with acronym NPA, chemical formula $\left(\mathrm{CH}_{3}\right)_{3} \mathrm{C}\left(\mathrm{CH}_{2} \mathrm{OH}\right)$ ], neopentylglycol [NPG, $\left.\left(\mathrm{CH}_{3}\right)_{2} \mathrm{C}\left(\mathrm{CH}_{2} \mathrm{OH}\right)_{2}\right]$, 2-amino, 2-methyl, 1,3 propanediol [AMP, $\left(\mathrm{NH}_{2}\right)\left(\mathrm{CH}_{3}\right) \mathrm{C}\left(\mathrm{CH}_{2} \mathrm{OH}\right)_{2}$ ], tris(hydroxymethyl)-aminomethane [TRIS, $\left.\left(\mathrm{NH}_{2}\right) \mathrm{C}\left(\mathrm{CH}_{2} \mathrm{OH}\right)_{3}\right]$, 2-methyl, 2-nitro propane [MN, [ $\left.\left(\mathrm{NO}_{2}\right) \mathrm{C}\left(\mathrm{CH}_{3}\right)_{3}\right]$ and 2-methyl, 2-nitro, 1-propanol [MNP $\left.\left(\mathrm{NO}_{2}\right)\left(\mathrm{CH}_{3}\right)_{2} \mathrm{C}\left(\mathrm{CH}_{2} \mathrm{OH}\right)\right]$. These compounds have been extensively studied $[1,2,9,24-28]$ but thermodynamic data under pressure are rather scarce.

Powdered NPA, NPG, MN, MNP (99\% purity) and TRIS (99.9\% purity), were purchased from Sigma-Aldrich, and used as such. Powdered AMP was purchased from Fluka with a purity of $99.5 \%$ and used as such. Experimental methods and devices used for X-Ray powder diffraction (XRPD), Differential Scanning Calorimetry at normal pressure (DSC), and High-Pressure Differential Thermal Analysis (HP-DTA) up to $300 \mathrm{MPa}$ are the same as those described elsewhere [9]. For the HP-DTA up to $600 \mathrm{MPa}$, a high-pressure cell model MV1-30 was acquired from the Institute of High Pressure Physics of the Polish Academy of Science (Poland), with a temperature range from $193 \mathrm{~K}$ to $393 \mathrm{~K}$. Here, the samples were encapsulated in the same way as used in the other HP-DTA cells whereas the calorimetric signal between sample and reference was obtained by attaching the encapsulated samples to a peltier module, instead of using thermocouples in the Bridgman's piston setup. Another peltier module was used for the reference (that was left empty), and both peltiers were connected in opposition. As a usual procedure in calorimetry, heating rates were approximately $2 \mathrm{~K} \mathrm{~min}^{-1}$, although in some extreme temperatures the rates could be different. Dilatometry measurements in isothermal conditions were performed in a custom-built device similar to that described in Reference [29], allowing pressures from 0 to $300 \mathrm{MPa}$.

Analysis of the voltage peaks in HP-DTA curves permits the evaluation of transition temperatures, and enthalpy and entropy changes. The latter two require calibration of the HP-DTA signal. On the one hand, the sensitivity of the calorimetric cell is calculated from HP-DTA analysis of the solid-to-solid transition characteristics of three $\mathrm{Cu}$-Al-based alloys, which are assumed to be insensitive to the application of hydrostatic pressure. On the other hand, the normal-pressure value obtaed in HPDTA is forced to match the value from DSC. Measurements obtained by XRPD and dilatometry render volume changes and related quantities such as isothermal compressibility and isobaric thermal expansion. 

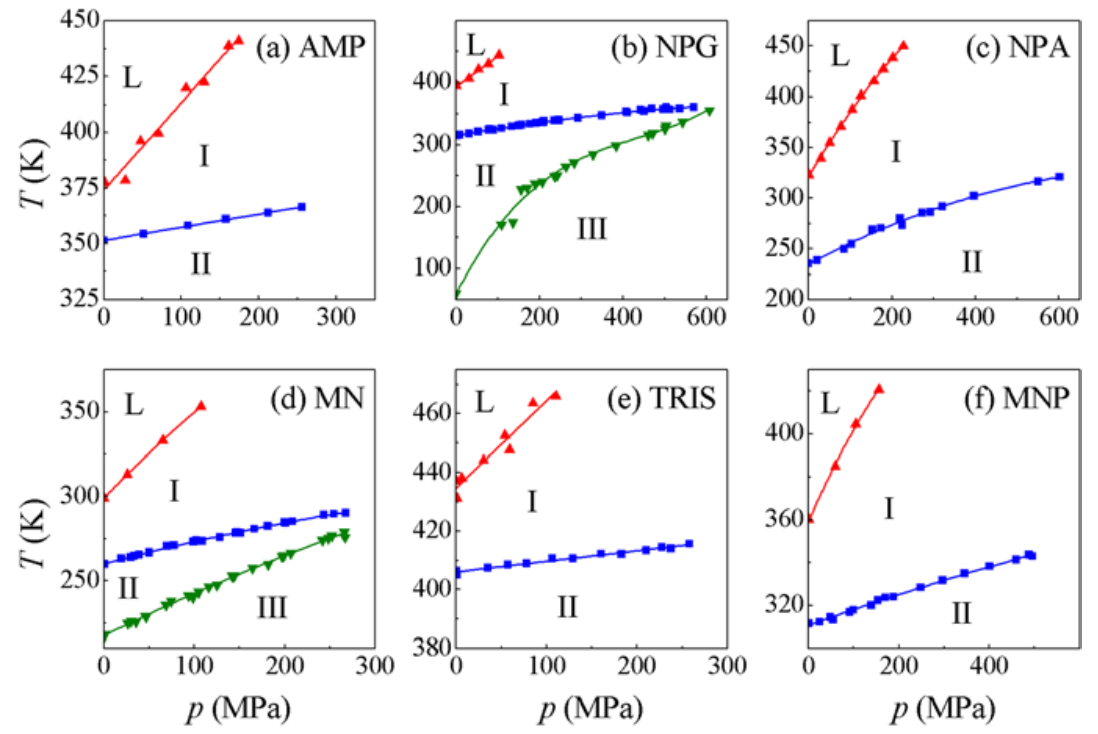

Fig. 1. T- $p$ phase diagrams for the neopentane derivatives. In all cases, there is an increase of the temperature range of stability of the plastic phase (denoted by I) as the pressure increases.

It is worth discussing about the error associated with the experimental methods used in this work. Although the precision of the techniques may be relatively high $\left(\varepsilon(T) \sim 1 \mathrm{~K}, \varepsilon(p) \sim 1 \mathrm{MPa}, \varepsilon(V) \sim 1 \mathrm{~cm}^{3} \mathrm{~mol}^{-1}\right)$, there are several major sources of error that decrease significantly the accuracy of the obtained results. In a very first step, the compound characteristics may depend on the particular sample, as the purity and the sample preparation does. In calorimetry experiments in alloys, for instance, it is known that the presence of inhomogeneities may result in anomalies in the calorimetric signal [30]. Moreover, here it is worth mentioning the error coming from the calibration process and the peak integration after baseline subtraction. This may explain the significant difference between the magnitude of thermodynamic quantities reported in the literature as, for instance, it is the case of the specific heat change $\Delta C_{p}$ across the plastic transition in NPG [31-33]. High-pressure experiments entail less accurate experimental data, with the presence of higher noise that complicates the choice of appropriate baselines. Moreover, some thermodynamic quantities require intermediate numerical steps (fits, derivatives, etc.) that may decrease dramatically the accuracy of the calculated magnitudes. For instance, here $\Delta \alpha^{*}(p)$ must be estimated as the difference between derivatives of numerical fits from thermal data obtained in dilatometry measurements. Hence, we can only assure relative errors $\varepsilon_{r}$ lower than or similar to $1 \%$ in $d T / d p$ and lower than $10 \%$ in $\Delta S_{0}$. For most of the remaining experimental magnitudes, $\varepsilon_{r}$ are estimated to be around $10-20 \%$ whereas in second-order terms, such as $\Delta \beta^{*}$ or $\Delta \alpha^{*}, \varepsilon_{r}$ can be much larger as discussed. Having said that, for the sake of clarity we will omit error bars in the figures.

\section{Results}

\section{Calorimetric results}

Figure 1 reports the temperature-pressure phase diagrams for the compounds under analysis obtained by HP-DTA. From now on, L, I, II and III will refer to liquid, 

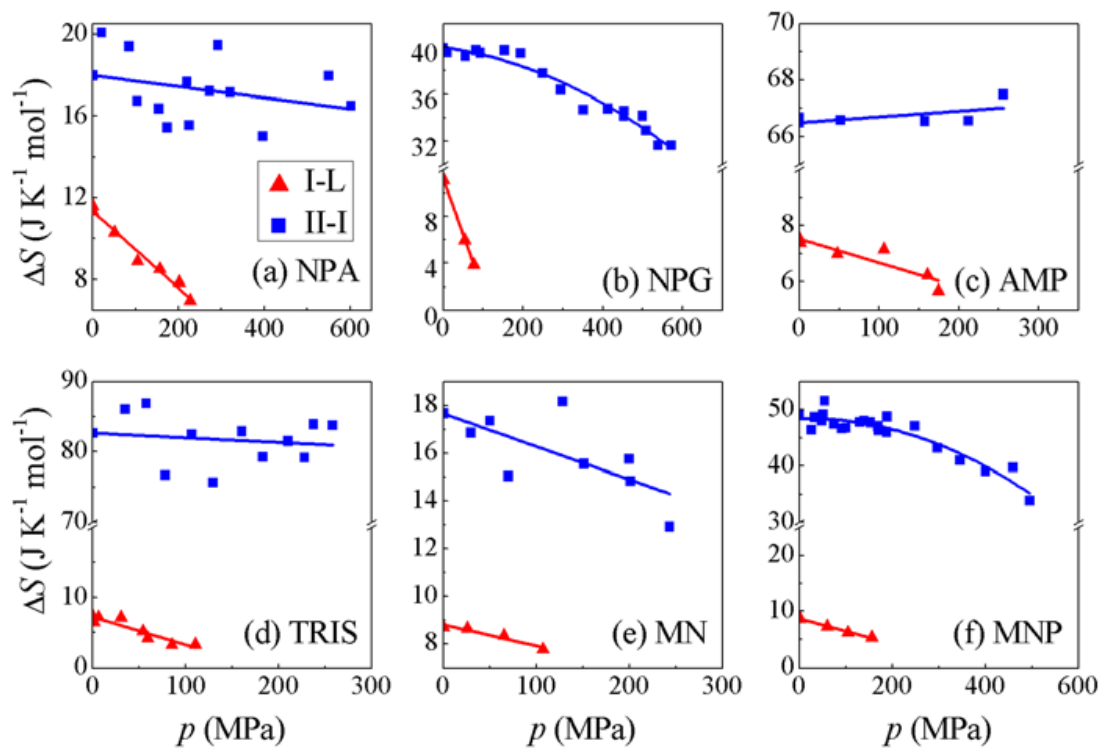

Fig. 2. Entropy change across the melting $\left(\Delta \mathrm{S}_{m}\right.$, red symbols) and plastic transitions $\left(\Delta \mathrm{S}_{t}\right.$, blue symbols) for the compounds under study as function of pressure. Lines are fits to the data.

plastic, and one and subsequent completely ordered crystalline phases respectively. Melting data for MN in this and following figures have been taken from literature [34] for completeness. Most of our data is consistent within error with literature data at normal pressure [1-4,35-37]. However, partial disagreement has been found in few cases [2] as for instance, the melting transition for TRIS, most likely due to less accurate measurement technique based on a metabolemeter, compared to that used here.

Figure 2 shows the molar entropy change across the melting $\left(\Delta S_{m}\right)$ and plastic $\left(\Delta S_{t}\right)$ transitions for each compound. Red (blue) lines and symbols indicate the melting (plastic) transition. It can be observed that in all cases the temperature range of stability of the plastic phase is enlarged when the pressure is increased $\left(d T_{m} / d p>d T_{t} / d p\right)$ and the entropy change on melting is lower than that associated to the plastic transition $\left(\Delta S_{m}<\Delta S_{t}\right)$. We recall that this behavior is common within compounds exhibiting plastic phases, as anticipated in the introduction.

We can use the data fits in the previous Figures 1 and 2 to compare the behavior between different compounds, as an attempt to extract any further universal behavior within plastic crystals. This is shown in Figure 3. Figure 3(a) shows $\Delta S_{m}$ as function of the parameter $T_{t} / T_{m}$, for different compounds. This helps the understanding of Figure 3(b), where the same data is plotted for different values of pressure. There, at each pressure (for a given color), each symbol stands for one compound. Linear regressions are carried out to detect trends in the limit of $T_{t} / T_{m} \rightarrow 1$. They reveal that, while at normal pressure $\Delta S_{m} \rightarrow \mathrm{R} \ln 2$, in agreement with the behavior recently reported in other sets of compounds [9], this trend is broken at high pressures.

Instead, Figures $3(\mathrm{c}, \mathrm{d})$ show that $d\left(T_{m}-T_{t}\right) / d p$ is quite similar for all the compounds and that $\Delta S_{t}$ approximately does not depend on the distance between transition temperatures, $T_{m}-T_{t}$. It is worth anticipating here that $\Delta V_{t}$ does change with pressure for all the studied compounds as it will be seen later on. It is consistent with the fact that the $T$ - $p$ curves for the plastic transitions exhibit non vanishing curvature, in agreement with Clausius-Clapeyron equation. 

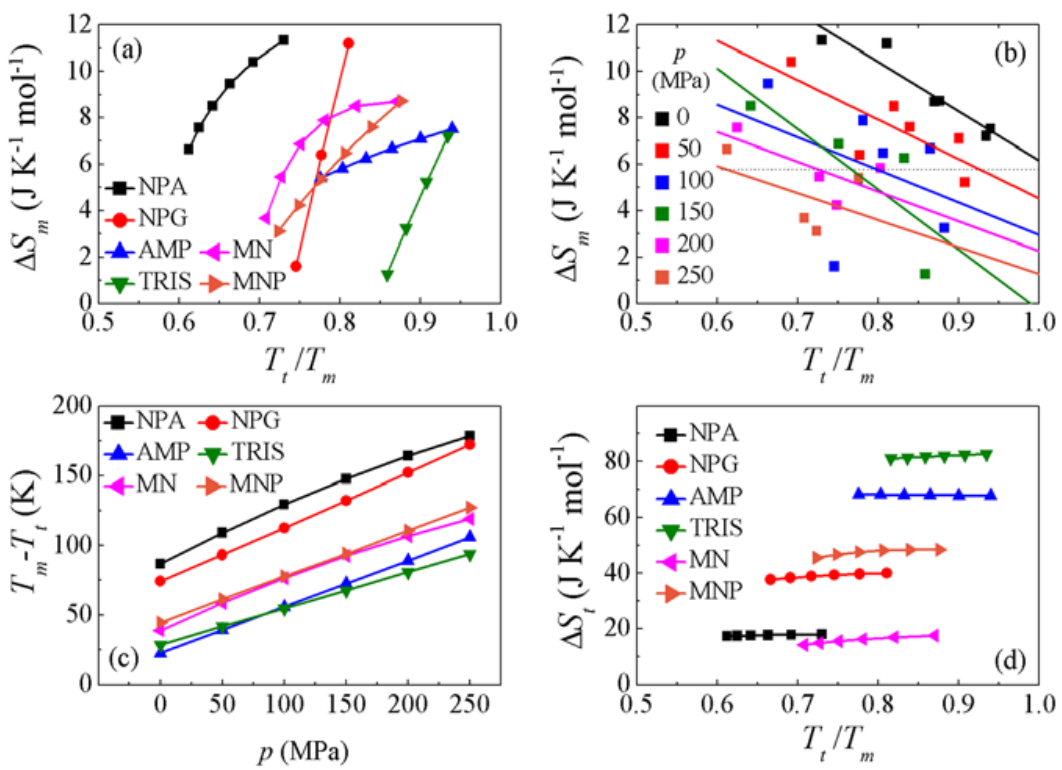

Fig. 3. (a) Entropy change on melting as function of $T_{t} / T_{m}$ for different samples. $T_{t}$ and $T_{m}$ stand for plastic and melting transition temperature respectively. (b) Same data as in (a), but color code corresponds to constant pressure. (c) $T_{m}-T_{t}$ as function of pressure for different samples. (d) Entropy change at the plastic transition as function of $T_{t} / T_{m}$ for different samples.

\section{Dilatometry, X-ray powder diffraction and Clausius-Clapeyron equation}

In this section we aim to test the Clausius-Clapeyron-related Equations (1-4) associated with the phase transitions in three of the compounds studied above, namely NPA, NPG and MN.In addition to calorimetric data presented above that provides the magnitude of $d T / d p$ and $\Delta S$ across the transitions, the knowledge of thermal coefficients is required. For this purpose we use dilatometry and XRPD data to measure the isothermal and isobaric evolution of volume respectively, which in turn will permit evaluating the thermal expansion $\alpha$ and isothermal compressibility $\beta$ in the different phases as well as $\Delta V$ across the transitions.

Figure 4 shows the evolution of the volume in temperature at normal pressure, obtained by XRPD. From these data, transition volume changes $\Delta V$ and thermal expansion $\alpha$ at either side of the transitions can be calculated.

Figure 5 shows high-pressure dilatometric experimental data for NPA, NPG and MN. While for NPA and NPG we use our own experimental data, for MN we use data previously published by Jenau et al. [34]. Large discontinuous changes correspond to phase transitions. The data have been fitted to a second order polynomial, for each phase separately. From Figure 5 we can then calculate the following Figures 6-10, where we have included data taken from Figure 4 when applicable. For instance, in Figure 6 we show the volume change $\Delta V$ across the different transitions, as function of pressure, including the value at normal pressure obtained from X-ray diffraction shown in Figure 4. From linear fits (straight lines in Figure 6) we can obtain the values for $d(\Delta V) / d p$. Figure 7 shows compressibility-related values, $\beta^{*}$ as function of pressure, obtained from the fits in Figure 5. Evaluation of this quantity close to each transition permits calculating $\Delta \beta^{*}=\beta_{i}^{*}-\beta_{j}^{*}$, where $i$ and $j$ stand for different phases at the transition, which is needed for Equations $(1,2,4)$ (see Figure 8). Figure 9 shows volume-temperature data as function of temperature extrapolated from the fits in 

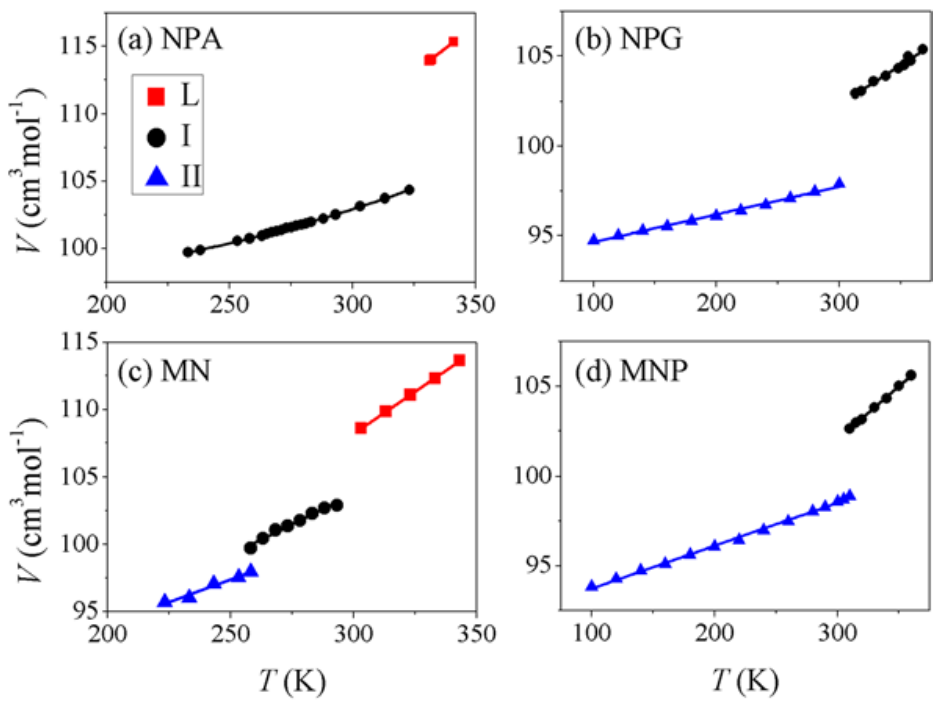

Fig. 4. X-Ray powder diffraction at normal pressure for four of the compounds under study: (a) NPA, (b) NPG, (c) MN and (d) MNP. Lines are fits to the data.
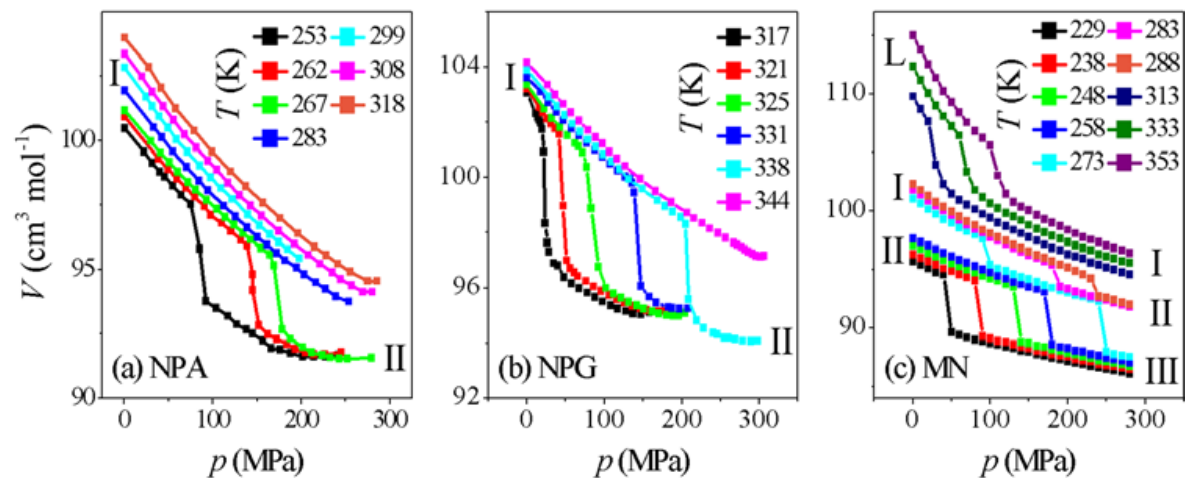

Fig. 5. Isothermal evolution of volume as function of pressure for NPA, NPG and MN. In the case of MN, the data is reproduced from Reference [34]. Phases L, I, II, and III are indicated near the corresponding regions.

Figure 5 for different applied pressures. Lines are linear fits to the data in all cases, as it is usually observed in organic crystals [7]. From the slopes of the linear fits, we can then extract the values for thermal expansion-related values, $\alpha^{*}$ as function of either temperature or pressure, the latter case shown in Figure 10.

Table 1 summarizes the data needed for Equations (1-4) for the different compounds. As we do not have complete data for all compounds, we will be able to evaluate the equations for NPA, NPG and MNP. Data taken from the literature is specified by different superscripts, and the corresponding references are indicated in the caption.

In Table 2 we compare the value for $d T / d p$ obtained directly from experiments with that of Clausius-Clapeyron equation at normal and high pressures. It is found that the agreement is excellent. Instead, comparison to Equation (1) fails completely, indicating that Equation (1) is only valid much closer to the transition, where the 

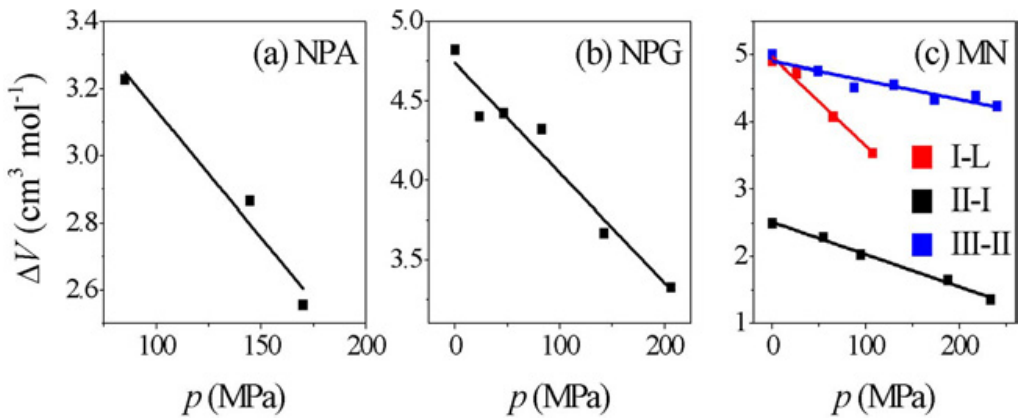

Fig. 6. Volume changes across the phase transitions as function of pressure. Values at normal pressure are taken from X-ray diffraction (see Fig. 4) whereas values under pressure are measured from dilatometry (see Fig. 5). The legend in panel (c) holds for all panels.
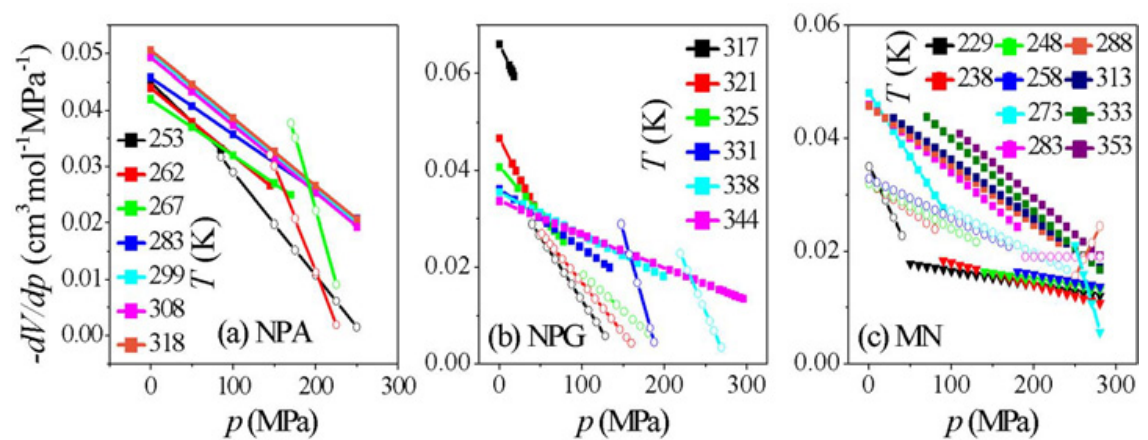

Fig. 7. Pressure derivative of volume as function of pressure, calculated from fits in figure 5. Filled squares, empty circles and downwards triangles stand for phases I, II and III respectively.
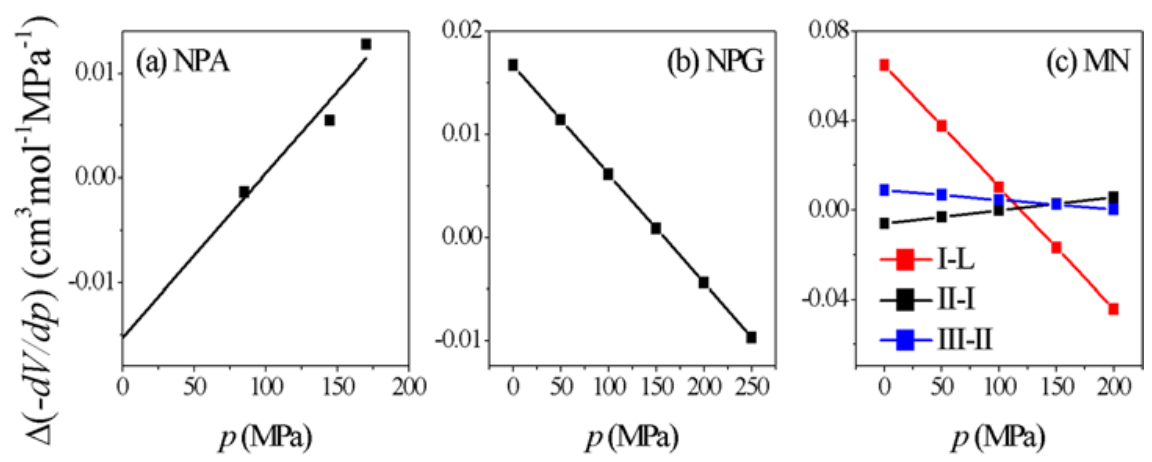

Fig. 8. Difference between pressure derivatives of volume at the transition between phases, calculated from the transition points of the fits in Figure 7.

magnitudes playing a role in the equation can be considered as constant, as it has been mentioned in the introduction. Indeed, Figures 6,8 and 11 show that this valid pressure range should be restricted to few MPa. The very significant disagreement also suggests that the errors associated with these magnitudes are likely too large for ${ }_{232}$ a reliable estimation. 

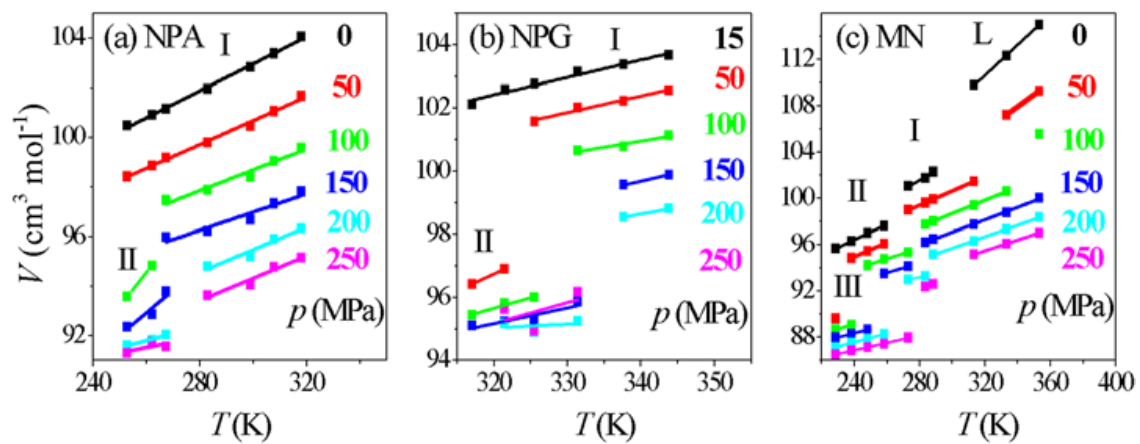

Fig. 9. Volume as function of temperature, extrapolated from Figure 5. Phases are indicated by L, I, II, III in the corresponding regions.
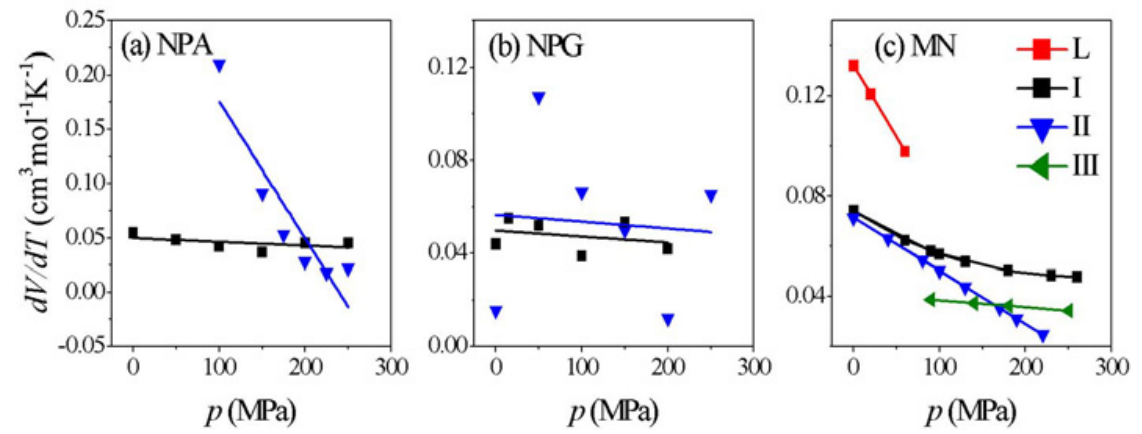

Fig. 10. Temperature derivative of volume $\left(\alpha^{*}\right)$ as function of pressure. Lines are fits to the data.
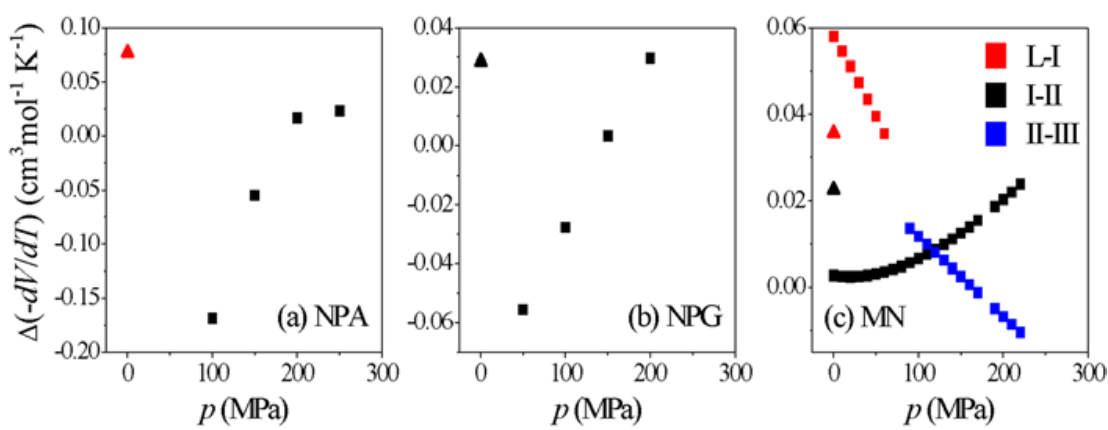

Fig. 11. Difference of $\alpha^{*}$ between phases, $\Delta \alpha^{*}$, as function of pressure. Square data have been calculated from data in Figure 10 whereas triangles are calculated from X-ray data (see Figure 4).

We then proceed to compare some thermodynamic quantities obtained through experiments with the corresponding values obtained using Equations (2-4) (see table 3 ). It is revealed that in general the latter values differ significantly from the former. Again, this discrepancy can be attributed to the increase of errors due to the accumulation of numerical steps necessary to infer indirectly some quantities that have not been possible to reach directly from experiments. As a consequence, this makes that the final calculated values are extremely sensitive to the initial experimental values. This is the case, for instance, of $\Delta \alpha^{*}$, that has been obtained through 
Table 1. Transition characteristics for the set of neopentane derivatives analyzed in this work. Subscript "0" refers to the values at or across the transition at normal pressure. "Taken from Reference [2]. ${ }^{\ddagger}$ Taken from Reference [32]. ${ }^{*}$ Taken from Reference [33]. ${ }^{\#}$ Taken from Reference [Kamae 31]. ${ }^{¥}$ Taken from Reference [38]. ?Taken from Reference [39].

\begin{tabular}{|c|c|c|c|c|c|c|c|}
\hline & & NPA & NPG & AMP & TRIS & MN & MNP \\
\hline \multirow{2}{*}{$T_{\mathrm{t}}$ at $0.1 \mathrm{MPa} \mathrm{K}$} & L-I & 323.2 & 394.5 & 374.1 & 437.1 & 299.0 & 360.2 \\
\hline & I-II & 235.7 & 314.8 & 351.4 & 406.1 & 260.6 & 311.6 \\
\hline \multirow{2}{*}{$\begin{array}{l}d T_{\mathrm{t}} / d p \text { at } 0.1 \\
\mathrm{KMpa}^{-1}\end{array}$} & L-I & 0.688 & 0.494 & 0.392 & 0.297 & 0.561 & 0.482 \\
\hline & I-II & 0.218 & 0.118 & 0.0590 & 0.0361 & 0.141 & 0.0750 \\
\hline \multirow{2}{*}{$\begin{array}{l}d^{2} T_{\mathbf{t}} / d p^{2} \\
\mathbf{1 0}^{-4} \mathbf{K} \mathbf{M p a}^{-2}\end{array}$} & L-I & -10.8 & 0 & 0 & 0 & -10.2 & -11.0 \\
\hline & I-II & -2.50 & -1.28 & 0 & 0 & -1.6 & -0.339 \\
\hline \multirow{2}{*}{$\begin{array}{l}\Delta S_{0} \\
\mathrm{~J} \mathrm{~K}^{-1} \mathbf{m o l}^{-1}\end{array}$} & L-I & 11.3 & 11.2 & 7.52 & 7.21 & 8.7 & 8.71 \\
\hline & I-II & 18.0 & 39.9 & 66.5 & 82.7 & 17.9 & 49.1 \\
\hline \multirow{2}{*}{$\begin{array}{l}\Delta V_{0} \\
\mathbf{c m}^{3} \mathbf{m o l}^{-1}\end{array}$} & L-I & $7.90^{\dagger}$ & $6.63^{\dagger}$ & $3.39^{\dagger}$ & $3.14^{\dagger}$ & 4.9 & $4.46^{\dagger}$ \\
\hline & I-II & $4.01^{\dagger}$ & 4.82 & $5.06^{\dagger}$ & $5.07^{\dagger}$ & 2.5 & $3.90^{\dagger}$ \\
\hline \multirow{2}{*}{$\begin{array}{l}\Delta c p 0 \\
\mathrm{~J} \mathrm{~K}^{-1} \mathrm{~mol}^{-1}\end{array}$} & L-I & $21.5^{\ddagger}$ & $20.4^{*}$ & $26.3^{*}$ & $5.81^{*}$ & $6.71^{¥}$ & \\
\hline & I-II & & $\begin{array}{l}64.4^{\ddagger} \\
68.9^{\#} \\
71.8^{*}\end{array}$ & $127^{*}$ & $\begin{array}{l}147^{\ddagger} \\
142^{*}\end{array}$ & $-12.1^{¥}$ & $44^{?}$ \\
\hline \multirow{2}{*}{$\begin{array}{l}\Delta \alpha_{0}^{*} \\
\mathbf{c m}^{3} \mathbf{m o l}^{-1} \mathbf{K}^{-1}\end{array}$} & L-I & 0.077 & & & & 0.036 & \\
\hline & I-II & & 0.029 & & & 0.023 & 0.035 \\
\hline \multirow[t]{2}{*}{$\begin{array}{l}\Delta \beta_{0}^{*} \\
\mathbf{c m}^{3} \mathbf{m o l}^{-1} \mathrm{MPa}^{-1}\end{array}$} & L- I & & & & & 0.065 & \\
\hline & I-II & -0.015 & 0.017 & & & -0.006 & \\
\hline \multirow{2}{*}{$\begin{array}{l}d \Delta H_{\mathbf{0}} / d p \\
\mathbf{J} \mathbf{m o l}^{-\mathbf{1}} \mathbf{M P a}^{-\mathbf{1}}\end{array}$} & L-I & -2.2 & -38 & -0.95 & -18 & 1.3 & -5.8 \\
\hline & I-II & 1.5 & 1.7 & 4.0 & -0.23 & -3.0 & 4.6 \\
\hline \multirow{2}{*}{$\begin{array}{l}d \Delta V_{\mathbf{0}} / d p \\
\mathbf{c m}^{\mathbf{3}} \mathbf{m o l}^{-1} \mathbf{M P a}^{-1}\end{array}$} & L-I & & & & & -0.013 & \\
\hline & I-II & -0.0076 & -0.0069 & & & -0.0048 & \\
\hline
\end{tabular}


Table 2. Comparison between experimental fits and calculated values for $d T / d p\left(\mathrm{~K} \mathrm{MPa}^{-\mathbf{1}}\right)$ at several pressures. For pressures higher than $0.1 \mathrm{MPa}$, calculations of eq. (1) are also considered.

\begin{tabular}{|l|l|l|l|l|}
\hline $\mathbf{p}(\mathrm{MPa})$ & & $\mathbf{N P A}$ & $\mathbf{N P G}$ & $\mathbf{M N}$ \\
\hline \multirow{3}{*}{5.1} & experimental & 0.218 & 0.118 & 0.141 \\
\cline { 2 - 5 } & Clausius-Clapeyron & 0.217 & 0.118 & 0.141 \\
\hline \multirow{3}{*}{100} & experimental & 0.206 & 0.112 & 0.131 \\
\cline { 2 - 5 } & Clausius-Clapeyron & 0.197 & 0.111 & 0.130 \\
\cline { 2 - 5 } & Equation (1) & 0.26 & 0.12 & 0.18 \\
\hline \multirow{3}{*}{200} & experimental & 0.193 & 0.106 & 0.122 \\
\cline { 2 - 6 } & Clausius-Clapeyron & 0.177 & 0.103 & 0.120 \\
\cline { 2 - 6 } & Equation (1) & 0.30 & 0.13 & 0.23 \\
\hline & experimental & 0.168 & 0.093 & 0.102 \\
\cline { 2 - 5 } & Clausius-Clapeyron & 0.136 & 0.088 & 0.105 \\
\cline { 2 - 5 } & Equation (1) & 0.39 & 0.14 & 0.35 \\
\hline
\end{tabular}

Table 3. Comparison between experimental and calculated values through Equations (1-4) for $d T / d p, d^{2} T / d p^{2}, d \Delta H / d p$ and $d \Delta V / d p$ for the II-I transition at normal pressure for NPA, NPG and MN.

\begin{tabular}{|c|c|c|c|c|}
\hline & & NPA & NPG & $\mathrm{MN}$ \\
\hline \multirow{2}{*}{$\begin{array}{l}d^{2} \mathrm{~T} / d p^{2} \\
\mathrm{~K} \mathrm{MPa}^{-2}\end{array}$} & experimental & $-2.5 \cdot 10^{-4}$ & $-1.3 \cdot 10^{-4}$ & $-1.6 \cdot 10^{-4}$ \\
\hline & Equation (2) & $8.3 \cdot 10^{-4}$ & $-3.1 \cdot 10^{-4}$ & $7.4 \cdot 1^{-4}$ \\
\hline \multirow{2}{*}{$\begin{array}{l}d \Delta H / d p \\
\mathrm{~J} \mathrm{~mol}^{-1} \mathrm{MPa}^{-1}\end{array}$} & experimental & 1.5 & 1.7 & -3.0 \\
\hline & Equation (3) & 4.0 & 3.9 & -5.7 \\
\hline \multirow{2}{*}{$\begin{array}{l}d \Delta V / d p \\
\mathbf{c m}^{3} \mathbf{m o l}^{-1} \mathbf{M P a}^{-1}\end{array}$} & experimental & $-7.6 \cdot 10^{-3}$ & $-6.9 \cdot 10^{-3}$ & $-4.8 \cdot 10^{-3}$ \\
\hline & Equation (4) & $15 \cdot 10^{-3}$ & $-13 \cdot 10^{-3}$ & $9.1 \cdot 10^{-3}$ \\
\hline
\end{tabular}

differences of derivatives of data which in turn are calculated by fitting experimental data. We can then conclude that in the absence of high-quality direct experimental data, Equations (1-4) do not seem useful to determine any thermodynamic quantity, as traditional Clausius-Clapeyron does. We therefore discarded to extend the calculations of these equations to higher pressures or other transitions. 


\section{Conclusions}

By means of calorimetry, thermometry, dilatometry and X-ray diffraction, we have characterized the melting of orientational degrees of freedom at high pressure in a series of neopentane derivatives. We found that these composition-related compounds show that (i) the slope of the differences between the $T-p$ plastic-liquid and solid II-plastic transitions, $d\left(T_{m}-T_{t}\right) / d p$, is roughly independent of the compound, and (ii) at low-pressure the entropy change at the solid II-plastic transition is almost independent of pressure, in contrast to the volume change at the transition, that decreases notably when increasing pressure. This results in slightly convex curvature of the $T-p$ solid II-plastic transition line in most of the compounds. This behavior is consistent with Clausius-Clapeyron equation evaluated at high pressures. The present work represents one of the very few complete studies on the pressure-temperature dependence of entropy and volume in organic plastic crystals, and shows that some universal behavior might emerge for plastic phases. It should inspire similar work in other plastic crystals to confirm the observed trends as a generality beyond neopentane derivatives.

This work was financially supported by the Spanish Government, project No. MINECO FIS2014-54734-P, and by the Generalitat de Catalunya, project No. 2014SGR-00581.

\section{References}

1. E. Murrill and L. Breed, THermochim. Acta 1, 239 (1970)

2. J.Ll. Tamarit, B. Legendre, J.M. Buisine, Mol. Cryst. Liq. Cryst. 250, 347 (1994)

3. D.K. Benson, W. Burrows, J.D. Webb, Sol. Energy Mater. 13, 133 (1986)

4. M. Barrio, J. Font, D.O. López, J.Muntasell, J.L1. Tamarit, Sol. Energy Mater. Sol. Cells, 27, 127 (1992)

5. T. Clark, M.A. McKervey, H. Mackle, J.J. Rooney, J. Chem. Soc., Faraday Trans. 1 (1974)

6. T. Clark, T. Mc. O. Knox, H. Mackle, M.A. McKervey, J. Chem. Soc., Faraday Trans. 173, 1224 (1977)

7. G.J. Kabo, A.A. Kozyro, M. Frenkel, A.V. Blokhin, Mol. Cryst. Liq. Cryst. 326, 333 (1999)

8. M.B. Charapennikau, A.V. Blokhin, A.G. Kabo, G.J. Kabo, J. Chem. Thermodynamics 35, $145(2003)$

9. J.Ll. Tamarit, I.B. Rietveld, M. Barrio, R. Céolin, J. Mol. Struc. 1078, 3 (2014)

10. J. Reuter, D. Büsing, J. Ll. Tamarit, A. Würflinger, J. Mater Chem. 7, 41 (1997)

11. M. Barrio, P. de Oliveira, R. Céolin, D.O. López, J.Ll. Tamarit, Chem. Mater. 14, 851 (2002)

12. Ph. Negrier, L.C. Pardo, J. Salud, J.Ll. Tamarit, M. Barrio, D.O. López, A. Würflinger, D. Mondieig, Chem. Mat. 14, 1921 (2002)

13. J.Ll. Tamarit, M. Barrio, L.C. Pardo, P. Negrier, D. Mondieig, J. Phys.: Condens. Matter. 20, 244110 (2008)

14. M. Barrio, J.Ll. Tamarit, Ph. Negrier, L.C. Pardo, N. Veglio, D. Mondieig, New J. Chem. 32, 232 (2008)

15. B. Parat, L.C. Pardo, M. Barrio, J.Ll. Tamarit, Ph. Negrier, J. Salud, D.O. López, D. Mondieig, Chem. Mater. 17, 3359 (2005)

16. R. Levit, M. Barrio, N. Veglio, J.Ll. Tamarit, Ph. Negrier, L.C. Pardo, J. SanchezMarcos, D. Mondieig, J. Phys. Chem. B 112, 13916 (2008)

17. Ph. Negrier, M. Barrio, J.Ll. Tamarit, N. Veglio, D. Mondieig, Cryst. Growth \& Des. 10, $2793(2010)$

18. M. Woznyj, F.X. Prielmeier, H.-D. Lüdemann, Z. Naturforsch. 39a, 800 (1984)

19. H.G. Kreul, R. Waldinger, A. Würflinger, Z. Naturforsch 47a, 1127 (1992) 
20. P.F. McMillan, Nature Mater. 6, 7 (2007). Benzene bridges under pressure.

21. L. Ciabini, M. Santoro, F.A. Gorelli, R. Bini, V. Schettino, S. Raugei, Nature Mater. 6, 39 (2007)

22. P.W. Bridgman, Phys. Rev. 6, 94 (1915)

23. W. Wagner, A. Saul, A. Pruss, J. Phys. Chem. Ref. Data 23, 515 (1994)

24. T. Hasebe, H. Chihara, Bull. Chem. Soc. Jpn. 59, 1141 (1986)

25. J. Salud, M. Barrio, D.O. López, J. Ll. Tamarit, X. Alcobé, J. Appl. Cryst. 31, 748 (1998)

26. J. Salud, D.O. López, M. Barrio, J.Ll. Tamarit, J. Mater. Chem. 9, 909 (1999)

27. K. Arvidsson, E.F. Westrum. Jr., J. Chem. Thermodyn. 4, 449 (1972)

28. K. Suenaga, T. Matsuo, H. Suga, Thermochim. Acta 163, 263 (1990)

29. R. Landau, A. Würflinger, Rev. Sci. Instrum. 51, 533 (1980)

30. E.K.H. Salje, Phase Transitions in Ferroelastic and Co-elastic Crystals (Cambridge University Press, Cambridge, UK, 1990)

31. R. Kamae, K. Suenaga, T. Matsuo, H. Suga, J. Chem. Thermodyn. 33, 471 (2001)

32. D. Chandra, W.-M. Chen, V. Gandikotta, D.W. Lindle, Z. Phys. Chem. 216, 1433 (2002)

33. S. Divi, R. Chellappa, D. Chandra, J. Chem. Thermodyn. 38, 1312 (2006)

34. M. Jenau, J. Reuter, J.Ll. Tamarit, A. Würflinger, J. Chem. Soc., Faraday Trans. 92, $1899(1996)$

35. M. Rittmeier-Kettner, G.M. Schneider, Thermochim. Acta 266, 185 (1995)

36. M. Barrio, D.O. Lopez, J.Ll. Tamarit, Ph. Negrier, Y. Haget. J. Mater. Chem. 5, 431 (1995)

37. J. Salud, D.O. López, M. Barrio, J.Ll. Tamarit, H.A.J. Oonk, P. Negrier, Y. Haget, J. Solid State Chem. 133, 536 (1997)

38. S. Urban, Z. Tomkowicz, J. Mayer, T. Waluga, Acta Phys. Pol. A48, 61 (1975)

39. J. Font, J. Muntasell, Mater. Res. Bull. 29, 1091 (1994) 